\title{
Generación automática de mallas triangulares con control de error y aplicaciones en la industria
}

Development of a computer system for the automatic generation of triangular meshes with error control and applications in industry

\author{
José Arzola Ruiz ${ }^{1}$ Maximiliano Asís López ${ }^{2}$, Jesús Espinola Gonzales² Y \\ VLADIMIR Rodríguez SABINO²
}

\section{RESUMEN}

Se propone un método de aproximación de un objeto 3D representado por superficies de Bézier; a través de mallas triangulares con control del error. El procedimiento realizado, se resume en tres etapas principales: i) digitalización del objeto 3D en formato IGES; ii) generación de un conjunto de puntos aleatoriamente en el dominio de la superficie; y iii) el mallado de la superficie que representa al objeto 3D, controlando el error con un modelo matemático obtenido en este trabajo y la implementación del algoritmo de mallado obtenido, en Visual Basic.NET para visualizar el resultado.

Palabras clave: superficie de Bézier; mallas triangulares; objeto 3D; formato IGES.

\begin{abstract}
It is described the mathematical foundations and algorithmic for the development of an approach program; which approaches an object 3D represented by a surface of Bezier; through triangular meshes with a control of made error. Firstly the object 3D are digitized in IGES format. Then a group of points are generated aleatorily in the domain of the surface. Finally the meshing of the surface that represents the object 3D is made, controlling the error with an mathematical model obtained in this work also the algorithm of meshing obtained is implemented, in Visual Basic.NET to visualize the result.
\end{abstract}

Keywords: Bézier surfaces; triangulate mesh; 3D object; IGES format.

1 Instituto Superior Politécnico José Antonio Echevarría. CUJAE. La Habana, Cuba.

2 Universidad Nacional «Santiago Antúnez de Mayolo». Huaraz, Perú. 


\section{ICHIKLLACHAW}

Imanawpis 3D kaqkunata rikapaayaanaapaq, Bézier kaqchaw rurashqata, rurayanqaatam willakayaamuu; mana alli kaqta malla triangularwan tinkutsiryan. Kima ruraychawmi kayta rurayaamurquu: i) kumputaduraman churayarquu tsay kaqta 3D, IGES kanqannaw. ii) churapaayarquu chusukunata mana akrashpa, llapan patsanaw kaqman; y iii) patsanaw kaqchaw chusukunaman mallan churapay, tsaychaw $3 \mathrm{D}$ kaq, mana alli yarqunanpaq huk matimatika ruraywan rikapashqa, niykur huk alguritmuta rurayarquu rikaana patsanpita. Llapan uryaakunata rikaanapaq NETta churapaayarquu.

Pushaq shimikuna: Bézierpa patsan; kimakuchu maallakuna; 3D kaqkuna; IGES imanawpis kaqkuna.

\section{INTRODUCCIÓN}

El Diseño Geométrico Asistido por Computador (CAGD) está relacionado con la representación, manipulación y diseño de objetos matemáticos mediante un computador, tales como curvas, superficies y sólidos (Farin, 1993; Pinto et al., 2003). Las herramientas matemáticas del CAGD son principalmente el análisis matemático, el análisis numérico, cálculo variacional, la geometría diferencial, etc. Las principales aplicaciones se encuentran donde se necesita métodos para describir matemáticamente los objetos que van a ser manufacturados por máquinas controladas por computador, como por ejemplo: en la industria aeronáutica (diseño de alas, fuselajes de un avión, etc.), automotriz (diseño de las partes de un auto), etc. Otras aplicaciones se encuentran en los gráficos por computador, visualización científica para describir los fenómenos físicos, geológicos, médicos, etc. (Foley et al., 1996; Pinto et al., 2003).

Para la representación gráfica de estos objetos matemáticos, como para su manejo de edición o modificación, no siempre resulta fácil trabajarlos en sus representaciones algebraicas. Una alternativa es trabajarlas mediante aproximaciones, aquí es donde aparece la idea de aproximar una superficie mediante una malla.

Las ideas básicas para la generación de mallas en geometrías generales pueden clasificarse en tres grandes grupos (Patrikalakis, 1989).

El primero que mencionaremos consiste en la modificación del dominio original por la remoción sucesiva de triángulos a partir de su borde. Esta idea es comúnmente conocida como método frontal o método del frente de avance, debido a la forma en que evoluciona la frontera del dominio durante el proceso de generación. El método frontal fue utilizado con éxito para la generación de mallas de elementos finitos en dos dimensiones, y ha sido extendido al caso tridimensional.

Otra alternativa a la generación de mallas consiste en generar puntos sobre el dominio de alguna manera, y luego conectarlos para formar una triangulación que satisfaga alguna propiedad de optimalidad, esta triangulación es conocida como Triangulación de Delaunay de un conjunto de puntos. 
Un tercer método se basa en la utilización de una cuadrícula que se superpone al dominio, las celdas exteriores se descartan, mientras que el resto se puede subdividir sucesivamente de acuerdo al grado de discretización deseado en cada región del dominio. Finalmente se dividen las celdas interiores en triángulos, de modo que se mantenga la compatibilidad con las celdas vecinas. Las celdas que intersecan a la frontera requieren un tratamiento especial (pueden triangularse, por ejemplo, utilizando el método frontal). Esta técnica es conocida como «método de árboles cuaternarios», debido a la estructura de datos implícitos en la subdivisión sucesiva de cada celda, y ha sido utilizado en generación de mallas de elementos finitos tanto en dos como en tres dimensiones.

Una de las técnicas más utilizadas para realizar el mallado de objetos es la técnica de Delaunay; es una técnica muy versátil, que permite asociar a una nube de puntos en 2 o 3 dimensiones un mallado con buenas propiedades geométricas (es decir, los triángulos o tetraedros son tan próximos a ser equiláteros cómo es posible siguiendo la distribución de puntos).

En el diseño geométrico se manipula el diseño de una curva o superficie por el cambio de los llamados puntos de control. Las superficies y curvas se construyen por polinomios de Bézier o B-splines, en ambos casos estas superficies o curvas se encuentran en la cápsula convexa de sus puntos de control. Las curvas pasan por lo general por el primer y último punto de control y las superficies son limitadas por curvas de Bézier o B-splines (Prautzsch et al., 2002; Rogers, 2001).

\section{MATERIALES Y MÉTODOS}

\section{Triangulación Delaunay}

Es la generación de puntos sobre el dominio y luego conectarlos para formar una triangulación que satisfaga alguna propiedad de optimalidad. Por ejemplo, puede hallarse la triangulación que maximice el mínimo de los ángulos de cada par de triángulos adyacentes. La condición del ángulo mínimo es equivalente a la del círculo circunscrito. El círculo que pasa por los tres vértices de cada triángulo no contiene a otros puntos de la triangulación. Esta propiedad se utiliza para la construcción de la triangulación Delaunay (Rogers, 2001). La utilización de triangulaciones Delaunay en la generación de mallas se remonta a los trabajos de Cavedish y puede extenderse al caso tridimensional.

Algoritmo para el Mallado

El algoritmo utilizado en este trabajo para el mallado del dominio paramétrico de la superficie de Bézier es el algoritmo de Triangulación de Delaunay y se construye de la siguiente manera:

Se ordena los puntos de datos según una clave compuesta primero por su coordenada $x$ y luego por su coordenada $y$. Esta ordenación tiene como fin disminuir los tiem- 
pos de búsqueda de los puntos que cumplan las condiciones de mínimas distancias o máximos ángulos.

Para la implementación del mallado del conjunto de puntos generados aleatoriamente en el dominio paramétrico se ha utilizado el tercer algoritmo de Delaunay pero con una pequeña modificación; es decir, el algoritmo queda así:

Paso 1: creación de un triángulo ficticio, el cual abarca todos los puntos generados, es decir, encierra el conjunto de puntos usando una triangulación ficticia.

Paso 2: avanza por incremento, insertando nuevos puntos a la triangulación existente.

Paso 3: por cada punto de la nube, buscar el triángulo que lo contiene, es decir la búsqueda es hecha por todos los triángulos cuyos círculos inscritos contienen el nuevo punto. Para ello se realiza los siguientes pasos:

a. Sea T el triángulo que contiene al punto $p$ a insertar. Unir $p$ con cada uno de los vértices del triángulo $T$, creando tres nuevos triángulos.

b. Por cada uno de los tres triángulos, generados al insertar un punto se realiza el test del círculo circunscrito; es decir, se legaliza las aristas.

Paso 4: una vez insertados todos los puntos se borra todos los lados del triángulo ficticio.

\section{RESULTADOS}

Control de error para el mallado

Esta propuesta se concentra en encontrar una estimación matemática que permita modelar los detalles finos de las superficies de los objetos; para ello, se propone un método para la generación de la Superficie de Bézier que incorpore en su construcción mallas triangulares, tal que se aproxime a la geometría de la superficie del objeto físico obtenido del fichero IGES, la generación de malla se realiza a partir de un conjunto de puntos desorganizados que se generan aleatoriamente en el dominio paramétrico de la superficie de Bézier del objeto original.

El modelo que se plantea en este trabajo para resolver el problema de aproximar la superficie de un objeto a través de mallas triangulares; es decir, el modelo matemático para controlar el error cometido al aproximar la superficie del objeto es el siguiente:

Dado un punto $\vec{P}_{i} \in S(u, v)$ de la superficie y un punto $\vec{P}$ cercano a la superficie $\mathrm{S}(\mathrm{u}, \mathrm{v})$, nuestro problema es calcular $\|S(u, v)-\vec{P}\|$ tal que sea mínimo.

Es decir, dado un punto $\vec{P}$ cercano a la superficie, el sistema a resolver es:

$$
\text { Minimizar } f(u, v)=\|S(u, v)-\vec{P}\|
$$


$\mathrm{Y}$ es equivalente a minimizar:

$$
f(u, v)=\|S(u, v)-\vec{P}\|^{2}
$$

Si igualamos las derivadas parciales a cero obtenemos:

Desarrollando tenemos:

$$
\left[\begin{array}{l}
\frac{\partial f}{\partial u}(u, v) \\
\frac{\partial f}{\partial v}(u, v)
\end{array}\right]=0
$$

$$
A=\left[\begin{array}{l}
2(S(u, v)-\vec{P}) \frac{\partial S(u, v)}{\partial u} \\
2(S(u, v)-\vec{P}) \frac{\partial S(u, v)}{\partial v}
\end{array}\right]=0
$$

Este es un sistema de ecuaciones no lineales y lo resolveremos utilizando el método de Newton-Rapson para un sistema no lineal.

Es decir, utilizaremos el siguiente algoritmo:

Paso 1: Tomar $\mathrm{k}=1$

Paso 2: Mientras $k \leq N$, hacer los pasos $3-7$

Paso 3: Calcular $A$ y el jacobiano $J$ en el punto $x=\left(u_{0}, v_{0}\right)$

Paso 4: Resolver el sistema para $y: J y=-A$

Paso 5: Tomar $x=x+y$

Paso 6: Si $\|y\|<$ tol, entonces salida de la solución aproximada

Paso 7: $k=k+1$

Paso 8: termina la iteración

Donde:

$N$ es el número de iteraciones.

tol es el error cometido al resolver el sistema.

$$
\begin{aligned}
& x=\left(u_{0}, v_{0}\right) \text { es el punto inicial. } \\
& J \text { es la matriz jacobiana y su expresión es: }
\end{aligned}
$$




$$
\begin{gathered}
J=2\left[\begin{array}{ll}
a & b \\
c & d
\end{array}\right] \\
a=\left(\frac{\partial S(u, v)}{\partial u}\right)^{2}+(S(u, v)-\vec{P}) \frac{\partial^{2} S(u, v)}{\partial u^{2}} \\
b=\frac{\partial S(u, v)}{\partial v} \frac{\partial S(u, v)}{\partial u}+(S(u, v)-\vec{P}) \frac{\partial^{2} S(u, v)}{\partial v \partial u} \\
c=\frac{\partial S(u, v)}{\partial u} \frac{\partial S(u, v)}{\partial v}+(S(u, v)-\vec{P}) \frac{\partial^{2} S(u, v)}{\partial u \partial v} \\
d=\left(\frac{\partial S(u, v)}{\partial v}\right)^{2}+(S(u, v)-\vec{P}) \frac{\partial^{2} S(u, v)}{\partial v^{2}}
\end{gathered}
$$

En este trabajo el punto $\vec{P}$ considerado como cercano a la superficie es obtenido calculando el centroide de cada triángulo del mallado realizado al objeto.

\section{Ejemplo de aplicación}

Para prueba del programa implementado se utiliza el fichero IGES que representa la capota del carro:

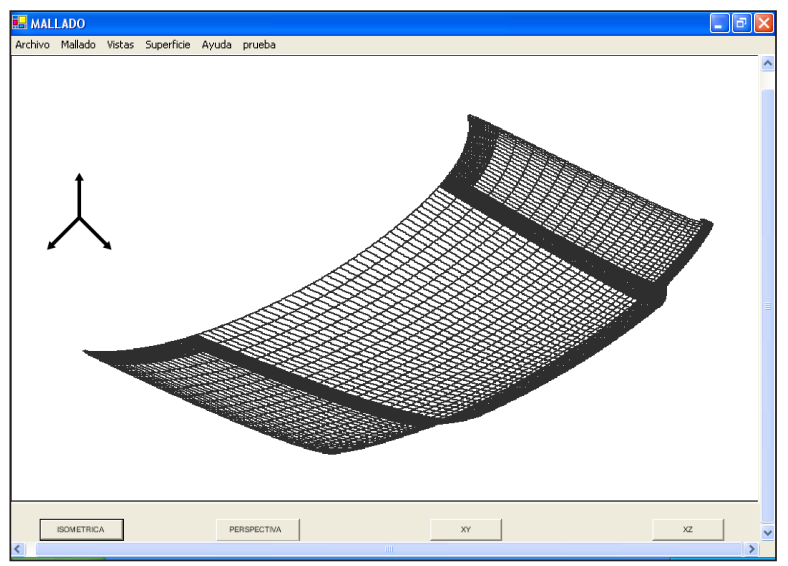

Figura 1. Visualización del archivo IGES del objeto vista Isométrica

Luego tomamos una sola superficie para realizar el mallado con el programa implementado. 


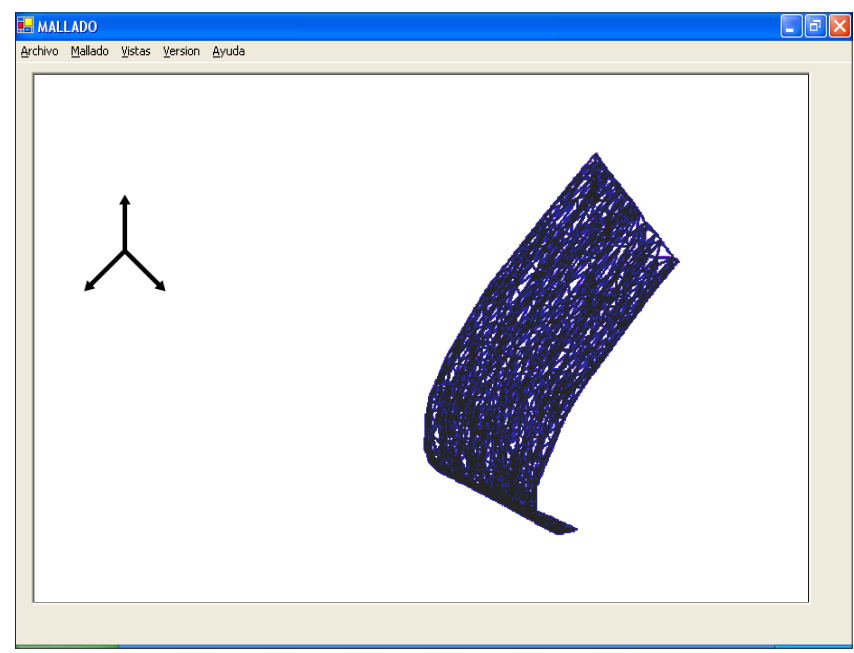

Figura 2. Visualización isométrica del mallado

\section{DISCUSIÓN}

\section{Elección del elemento para el mallado}

El número de posibles mallados sobre un conjunto de datos es muy grande. La selección del más adecuado se hace mediante criterios de optimización.

La elección del triángulo como elemento base de construcción de mallas aproximantes a las superficies, se debe a las propiedades importantes que tiene el triángulo.

Entre propiedades que tiene el triángulo como elemento de la malla, podemos mencionar las siguientes:

- El triángulo es el polígono con menor número de vértices.

- Queda definido completamente por las longitudes de sus lados, las cuales además deben cumplir con la desigualdad triangular.

- El triángulo es el polígono geométricamente indeformable y es además, necesariamente, convexo.

- La proyección de un punto interior sobre el perímetro es monótona, lo que quiere decir que los puntos proyección sobre el perímetro están ordenados y su relación de orden es del ángulo de las rectas proyectantes.

- Al ser el polígono más simple es imposible descomponerlo en varios y por lo tanto es necesariamente conexo.

- Un triángulo siempre define un interior y un exterior al mismo.

- El triángulo es el que presenta una mayor relación entre su perímetro y su área, con respecto a otros polígonos regulares.

- De todos los triángulos con un perímetro dado, el triángulo equilátero es el que hace mínimo el perímetro frente a su área.

- Como el triángulo equilátero es a la vez equiángulo; en este trabajo se impone que los elementos del mallado sean lo más equiláteros o equiángulos posibles. 
Por las razones antes mencionadas, en este trabajo se eligió hacer el mallado con el criterio de Delaunay, el cual optimiza la malla bajo la condición de maximizar los ángulos de los triángulos formados.

2. Ventajas del método de Delaunay

- Gran velocidad por el número de operación. El número de operaciones por elemento es menor debido a que en la creación del elemento por este método solo se compara círculos con puntos, en contraposición al avance frontal donde se realiza el cálculo de gran número de intersecciones.

- Siempre se obtiene una malla. Independiente de la calidad de los elementos, siempre se llega al final con este método, en contraposición al avance frontal donde puede llegarse a situaciones de no avance.

- Especialmente útil en el caso de que se disponga a priori de los nodos de la futura malla y únicamente se quiera crear los elementos.

\section{Elección del Lenguaje de Programación}

En este trabajo, la elección del lenguaje de programación ha quedado determinada, en gran parte, por el objetivo que tiene la aplicación y por las características que presenta el lenguaje. Para el desarrollo de la aplicación se ha elegido el lenguaje Visual Basic. NET.

Para el desarrollo de aplicaciones con interfaz gráfica amigable al usuario, es imprescindible elegir un lenguaje de programación Visual. De este modo se cuenta con la ventaja de que los elementos utilizados para la comunicación con la aplicación son ampliamente conocidos (ventanas, botones, menús, etc.) y fáciles de incorporar en la aplicación.

Además, es deseable que el proceso de implementación estuviese acorde con la apariencia moderna del lenguaje. Es decir, que se tratase de un lenguaje de programación orientado a objetos para aprovechar las ventajas de este tipo de metodología de programación (herencia, polimorfismo y encapsulamiento).

Por último, la elección de Visual Basic.Net como lenguaje de programación se debe a que dicho lenguaje se encuentra integrado con otros lenguajes de Microsoft Visual Studio.NET. No solo permite desarrollar componentes de aplicaciones en distintos lenguajes de programación, sino que también permite heredar las clases escritas en otros lenguajes.

\section{CONCLUSIONES}

Se plantea un modelo matemático adecuado a partir de las superficies de Bézier, para el control de error en la aproximación de objetos 3D a través de mallas triangulares. 
Se desarrolló un algoritmo que permite generar mallas automáticamente para superficies de Bézier controlando el error. Esto último es muy importante en las aplicaciones industriales donde se aplica el método de elementos finitos.

Se creó un software con prestaciones gráficas que implementa el algoritmo, desarrollado en este trabajo, que realiza el mallado con control de error a partir de superficies de Bézier.

La aplicación desarrollada lee la información geométrica de las superficies desde ficheros en formato IGES. Realiza el proceso de mallado, y presenta el resultado de forma gráfica; también se puede obtener los resultados numéricos.

El mallado que se obtiene es de tipo conforme con control de error, por lo que es muy importante para procesos de simulación en la industria, donde se usa el método de los elementos finitos.

El mallado de objetos bidimensionales y tridimensionales es un tema de gran importancia en muchas aplicaciones pero se utiliza generalmente para los siguientes propósitos:

La visualización de un objeto en un ordenador se manifiesta en base a los triángulos o tetraedros, por lo tanto, en general cualquier objeto para poder ser visualizado requiere previamente ser dividido (mallado) utilizando estas primitivas.

Respecto al diseño industrial, para diseñar puentes, aviones, carros, barcos, etc., es muy útil tener un modelo de objeto en tres dimensiones que se utilice para simular en un ordenador el comportamiento físico del modelo.

\section{REFERENCIAS BIBLIOGRÁFICAS}

Farin, Gerald. 1993. Curves and Surfaces for Computer Aided Geometric Design. A Practical Guide. San Diego: Academic Press, Inc.

Foley, James y otros. 1996. Computer Graphics: Principles and Practice. San Francisco: Addison-Wesley.

Patrikalakis, Nicholas.1989. Aproximate Conversion of Rational Splines. Computer Aided Geometric Design. Chicago: Elsevier Science Publishers.

Pinto, Carvalho; Figueiredo, Luis y Gomes, Jonas. 2003. Mathematical Optimization in Graphics and Vision. Lima: Monografías del IMCA.

Prautzsch, Hartmut; Boehm, Wolfgang y Paluszny, Marco. 2002. Methods of Bézier and B-Spline. Berlin: Springer-Verlag.

Rogers, David y Adams, James. 1990. Mathematical Elements for Computer Graphics. New York: McGraw Hill. 
|| José Arzola, Maximiliano Asís, Jesús Espinola y Vladimir Rodríguez

Rogers, David. 2001. An Introduction to Nurbs. San Diego: Academic Press San Diego, Annapolis.

Fecha de recepción: 12 de noviembre 2015

Fecha de aceptación: 07 de diciembre 2015

\section{Correspondencia}

José Arzola Ruiz

jararzola@ceter.cujae.edu.cu 